\title{
Impedance optimization and measurements of the injection stripline kicker
}

\author{
N. Wang, ${ }^{1, *}$ S. K. Tian $\odot,{ }^{1}$ L. Wang $\odot,{ }^{1}$ H. Shi $\odot,{ }^{1}$ S. Yue $\odot,{ }^{1,2}$ G. W. Wang, ${ }^{1}$ and J. H. Chen $\oplus^{1,2}$ \\ ${ }^{1}$ Key Laboratory of Particle Acceleration Physics and Technology, Institute of High Energy Physics, \\ Chinese Academy of Sciences, Beijing 100049, China \\ ${ }^{2}$ University of Chinese Academy of Sciences, Beijing 100049, China
}

(Received 31 August 2020; accepted 8 March 2021; published 24 March 2021)

\begin{abstract}
Stripline kickers are commonly used for on-axis injection in the low emittance rings. The beam coupling impedances of the kickers normally show large contributions to the total impedance budget. In the High Energy Photon Source, the necessity of the short pulse bottom width (less than $10 \mathrm{~ns}$ ) and strong deflection field requires the kicker to have a short length and a small gap between the electrodes, respectively. A new five-cell stripline kicker is proposed in order to save longitudinal space as well as to reduce the beam coupling impedance. Comprehensive studies have been undertaken to characterize the impedance of the stripline kicker. The beam coupling impedance and heat load dissipation on the kicker due to the beam passage are studied numerically. Strategies to further reduce the impedance are discussed. In addition, impedance bench measurements are launched to identify the impedance of the kicker experimentally. The longitudinal beam coupling impedance is measured with different terminations by using coaxial wire method. A satisfactory agreement has been reached between the numerical predictions and the measurements. A novel fasten block design with ceramic is proposed for the wire stretching and the center holding of the inner conductor in a small beam pipe.
\end{abstract}

DOI: 10.1103/PhysRevAccelBeams.24.034401

\section{INTRODUCTION}

A new generation of storage ring based light sources has been proposed in recent years to reach diffraction limited emittance of tens of pm [1-5]. The realization of the low emittance requires strong quadrupoles and sextupoles, which will result in small dynamic apertures (DAs). Therefore, the perturbations introduced by the injection system need to be kept small to avoid beam losses during injection. Different injection schemes [6-10] are proposed to relieve the requirements on the limited DAs. Stripline kickers are good candidates for their good impedance matching to the electrical circuit and low influence on the pulse signals.

Many accelerator facilities are developing their own stripline kickers to meet the physical requirements [11-19]. Among the most important considerations during the design of the stripline kicker are the beam coupling impedance and the beam energy deposition in the electrodes or the terminations during the beam passage. Many efforts have been undertaken to reduce the beam coupling impedance and beam-induced heat load $[11,12,20]$.

\footnotetext{
*wangn@ihep.ac.cn
}

Published by the American Physical Society under the terms of the Creative Commons Attribution 4.0 International license. Further distribution of this work must maintain attribution to the author(s) and the published article's title, journal citation, and DOI.
The High Energy Photon Source (HEPS) [1] is an ultralow emittance storage ring with beam energy of $6 \mathrm{GeV}$ and design beam current of $200 \mathrm{~mA}$. A multibend achromat lattice has been adopted to reach an ultralow natural emittance of $34.2 \mathrm{pm}$ with a circumference of $1360.4 \mathrm{~m}$. On-axis swap-out injection is adopted considering the limited DA and to reach top-up operation. With a fundamental $\mathrm{rf}$ frequency of $166.6 \mathrm{MHz}$, the beam has a minimum bunch spacing of $6 \mathrm{~ns}$, and an ultrafast kicker system with a pulse bottom width of less than $10 \mathrm{~ns}$ is required. The necessity of the short bottom width requires the kicker to have short electrodes. Meanwhile, a considerably large number, as well as small gap between the electrodes, are required to achieve a strong deflection field for the high energy beam.

Due to the restricted beam pipe dimensions and discontinuities at both ends of the electrodes, the stripline kicker can generate nontrivial beam coupling impedances. On the other hand, the electromagnetic field generated during the beam passage can induce heat load on the electrodes and propagate through the feedthroughs to the resistive terminators. Therefore, the beam coupling impedance must be well controlled during the design of the stripline kicker. The specifications for the beam coupling impedance are $Z_{y} \leq 50 \mathrm{k} \Omega / \mathrm{m}$ in the transverse plane and $Z_{\|} / n \leq 1 \mathrm{~m} \Omega$ in the longitudinal plane, which are $5 \%$ and $1 \%$ of the total impedance allowance, respectively. The heat load dissipated on each electrode needs to be less than $5 \mathrm{~W}$ 
(a)

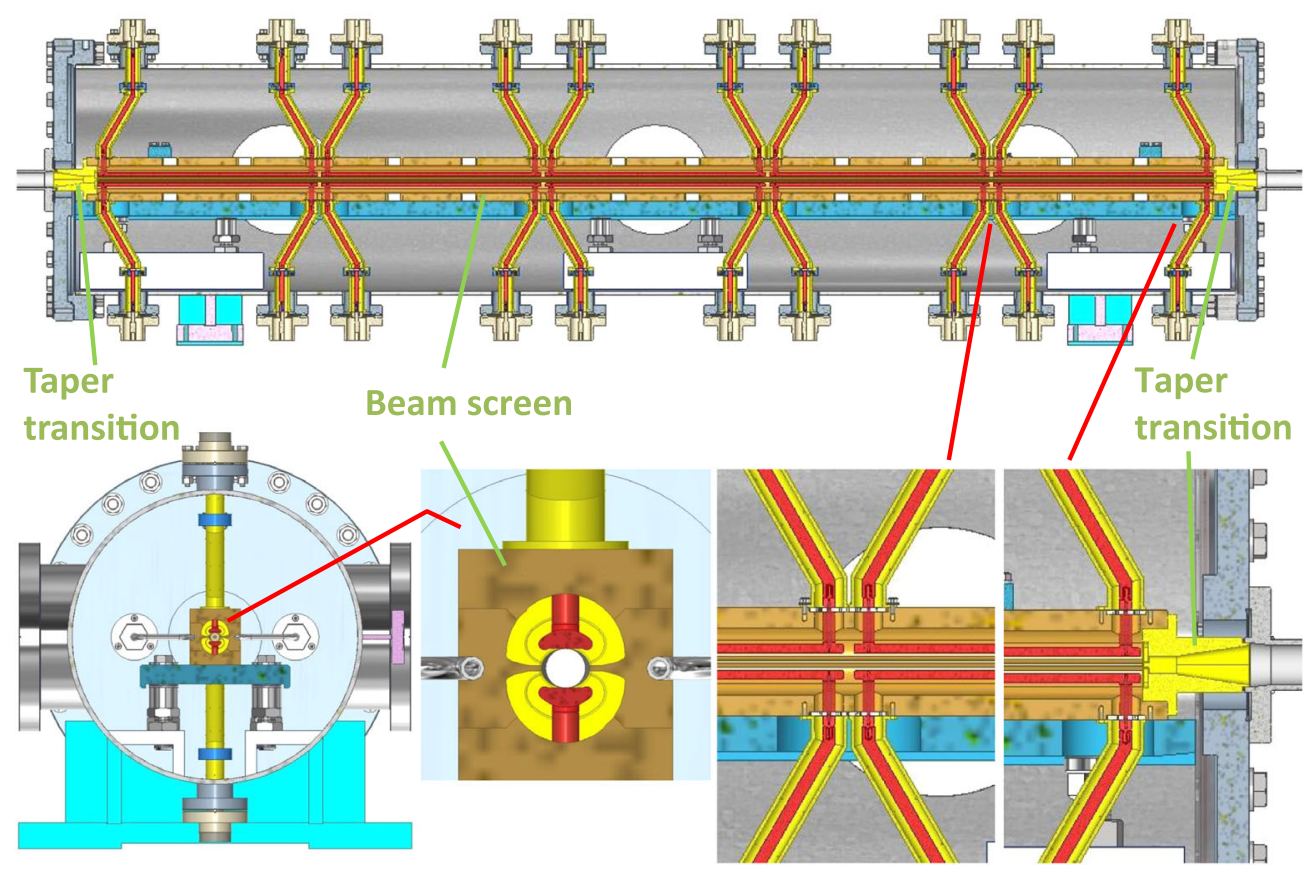

FIG. 1. CAD drawing of the five-cell stripline kicker: (a) longitudinal cross section along the beam axis, (b) transverse cross section in the middle of the stripline kicker and zoomed view of each end of the first set of striplines.

in order to avoid any heating issues. To meet the requirements, a novel five-cell stripline kicker, with five identical cell kickers in one vacuum module, is designed and fabricated [21].

The beam coupling impedance and heat load dissipation on the kicker are first studied numerically. The results are compared with those of five, individual, single cell kickers, demonstrating that the multicell kicker offers remarkable advantages in reducing the beam coupling impedances, since the discontinuity and resonances of the striplines are mitigated. Other strategies to further reduce the impedances are also discussed. On the other hand, the feedthroughs and terminations are not ideal. Normally, the transmission of the terminations and feedthroughs are frequency dependent, which will introduce reflections to the beam coupling impedance. Therefore, in order to characterize the impedance of the stripline kicker experimentally, as well as to identify the influence of the nonideal matching from the feedthroughs and the terminations, coaxial bench measurements have been carried out and benchmarked with numerical simulations. During the measurements, in order to hold and stretch the conducting wire at the center of the small beam pipe, a novel fasten block design with ceramic is proposed.

The paper is organized as follows. Section II describes the design features of the stripline kicker. Section III discusses the numerical estimations of the kicker impedance and beam energy dissipations on the electrodes and terminations. Strategies to reduce the beam coupling impedance are also investigated. Section IV discusses bench measurements of the beam coupling impedance with the coaxial wire method. The results are compared with numerical estimations, and satisfactory agreements have been reached. The conclusions are presented in Sec. V.

\section{DESIGN OF THE STRIPLINE KICKER}

The required bend angle of the kicker system at the design beam energy is $1.61 \mathrm{mrad}$. With an electrode length of $300 \mathrm{~mm}$, we need a pulse voltage of $17.1 \mathrm{kV}$ for each electrode and five stripline cells to provide the kick angle. The CAD drawing of the five-cell stripline kicker is shown in Fig. 1. Five kicker cells are installed in series with a longitudinal separation of $6 \mathrm{~mm}$ inside one vacuum tank. Each kicker cell has two " $\mathrm{D}$ " shaped electrodes placed in parallel with a vertical separation of $10 \mathrm{~mm}$. The electrodes are hosted inside an elliptical beam screen with two vanes introduced to decouple the electromagnetic field between the electrodes, which are essential in lowering the even mode characteristic impedances or beam coupling impedances [18-20]. Both the electrodes and the beam screen are made of copper to reduce the heat load dissipations from the beam. Multiple vacuum holes are implemented on the beam screen, together with the pumps on the outer vacuum tank, in order to reach high vacuum. There are four dedicated feedthroughs for each kicker cell to transfer power from the pulsers to the electrodes, where it is ultimately absorbed by the matched loads. Taper transitions are added at both ends of the kicker train to ensure smooth transition from the kicker to the ring beam pipe, and therefore to reduce the beam coupling impedance. 
The design of the five-cell stripline kicker offers the following remarkable advantages: (a) conserving the longitudinal space occupied by the kickers, since the flanges and the taper transitions between adjacent kickers can be omitted; (b) easing the vacuum pumping in the small vacuum chamber without occupying extra longitudinal space; and (c) reducing the beam coupling impedance, since the discontinuity and resonance of the striplines are mitigated, which will be discussed extensively in Sec. III.

\section{IMPEDANCE SIMULATIONS AND OPTIMIZATIONS}

Numerical simulations are performed, using CST STUDIO SUITE [22], to obtain the complete information regarding the beam coupling impedance and to evaluate the heat load dissipation due to the beam parasitic power loss. Different strategies to reduce the beam coupling impedance, as well as the heat load, are discussed.

\section{A. Simulation setups}

A simplified model with ideal coaxial feedthroughs has been used in the numerical studies. Taper transitions from the stripline kicker to the ring beam pipe are included. Here, the electromagnetic fields generated by the beam are supposed to be well shielded by the beam screen, and the outer vacuum tank is omitted.

The wakefields and impedances are calculated using Gaussian beams with an $\mathrm{rms}$ bunch length of $3 \mathrm{~mm}$. To study the resonant behavior of the stripline kicker, the wakefields are tracked over approximately 30 meters behind the bunch until the wakefields completely decay. The finite conductivity of the beam screen and the electrodes are included to evaluate the power loss dissipations and to determine reasonable $Q$ of the resonances.

\section{B. Beam coupling impedance}

The longitudinal and transverse beam coupling impedance of the five-cell stripline kicker up to $34 \mathrm{GHz}$ are shown in Figs. 2 and 3, respectively. The transverse electromagnetic (TEM)-mode-like resonances are observed below $\sim 4 \mathrm{GHz}$ with a frequency interval of $500 \mathrm{MHz}$, which corresponds to twice the length of the kicker cell. This is mainly determined by the resonant structure formed between the electrodes and the beam screen. At frequencies above $4 \mathrm{GHz}$, the impedances show no more quadratic sinusoidal wave dependence, since the electromagnetic field generated in the stripline will propagate with the beam [23]. The impedances are also compared with those of the single cell kicker, which have been multiplied by a factor of 5. The impedances of the single cell kicker show high order modes above $4 \mathrm{GHz}$, where several transverse modes already exceed the impedance threshold determined by the radiation damping. However, impedances are largely reduced for the five-cell kicker at frequencies above $4 \mathrm{GHz}$

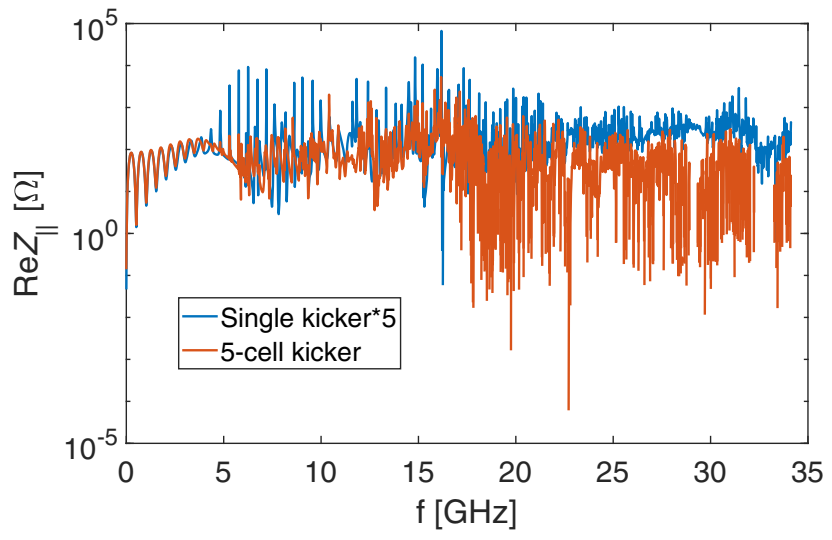

FIG. 2. The real part of the longitudinal beam coupling impedance of the five-cell stripline kicker, which is compared with that of the single cell kicker multiplied by a factor of 5 .

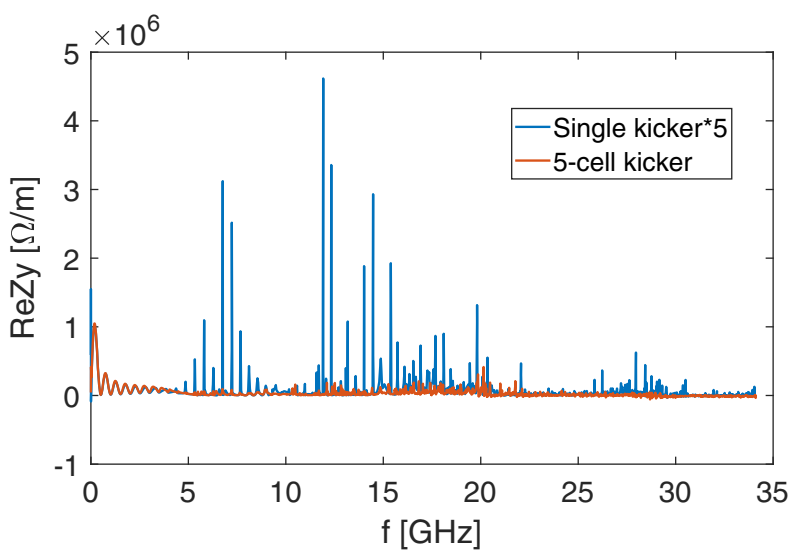

FIG. 3. The real part of the transverse beam coupling impedance of the five-cell stripline kicker, which is compared with that of the single cell kicker multiplied by a factor of 5 .

due to the mitigation resulting from the interaction among adjacent kicker cells.

The effective broadband impedances are also compared between the two cases. Both the longitudinal and transverse effective impedances are remarkably reduced for the fivecell kicker in a wide bunch length interval, from 3 to $30 \mathrm{~mm}$. This proves that the resonant structure formed by the electrodes and the beam screen become less inductive when multiple kicker cells are connected in series. This effect is more promising for short bunches, e.g., with a test bunch length of $3 \mathrm{~mm}$, both the loss factor and the transverse kick factor are reduced by a factor of $\sim 2$ compared to the single cell kicker (multiplied by 5 ), while the longitudinal effective impedance $Z_{\|} / n$ [24] is reduced by a factor of $\sim 10$. The specifications on the longitudinal and transverse impedances can only be met with the multicell design.

\section{Heat load dissipation due to the beam passage}

With a Gaussian test bunch of rms bunch length of $3 \mathrm{~mm}$, the heat load dissipation in the stripline kicker and beam 


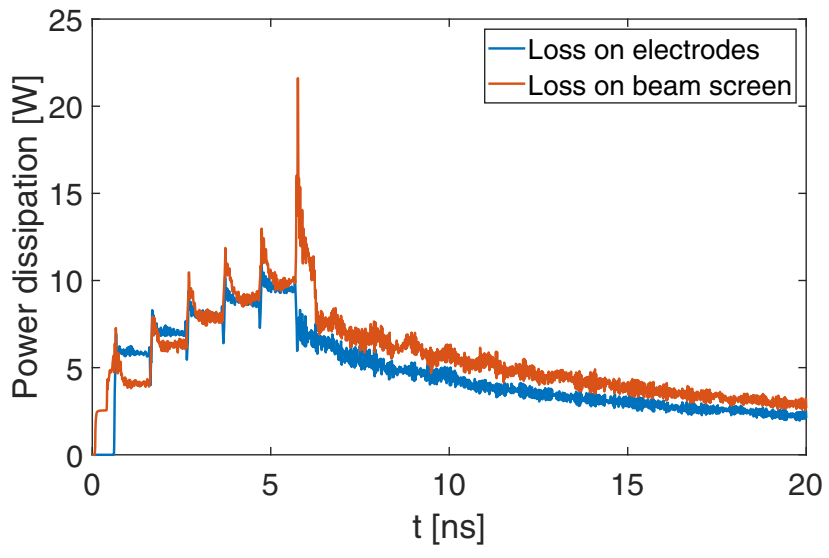

FIG. 4. Power dissipation on the electrodes and the beam screen during and after the bunch passage with bunch charge of $1 \mathrm{nC}$.

induced voltage on the feedthroughs are estimated. Considering a total beam current of $200 \mathrm{~mA}$ with bunch number of 680 , the loss factor corresponds to a total parasitic power loss from the beam of approximately $760 \mathrm{~W}$. Here, we only take this parameter setting as an example and omit the discussion regarding the different operation scenarios.

With the wakefield solver in CST, the power losses dissipated on the electrodes and the beam screen after the passage of one bunch of $1 \mathrm{nC}$ are recorded, as shown in Fig. 4. The peaks indicate that higher power losses occur when the beam passes through the gap between the electrodes. The power dissipation decreases gradually after the bunch passage.

The total power loss $P_{\text {loss }}$ when considering $N_{b}$ bunches uniformly filled in the ring can be estimated as follows:

$$
P_{\mathrm{loss}}=N_{b}\left(\frac{N_{e}}{N_{c}}\right)^{2} f_{\mathrm{rev}} \int_{0}^{t} P_{c}(t) d t
$$

where $N_{e}$ is the charge of one single bunch, $f_{\text {rev }}$ is the revolution frequency of the beam, $N_{c}$ is the charge of the exciting bunch in the wakefield simulations, and $P_{c}$ is the power loss recorded in unit time intervals, as given in Fig. 4. The results show a total power loss of $39 \mathrm{~W}$ dissipated on the electrodes and $48 \mathrm{~W}$ dissipated on the beam screen, which are well below the heat load specifications and less than half of that lost in a single cell kicker.

The other part of the parasitic power loss mainly transports to the upstream and downstream feedthroughs, and is ultimately absorbed by the terminations. This part can be calculated from the voltage signal $V_{\text {port }}$ induced by the beam at the feedthrough ports.

The beam power passing through the feedthroughs can also be estimated by Eq. (1) with $P_{c}(t)$ replaced by $V_{\text {port }}(t) / R_{0}^{2}$, where $R_{0}$ is the characteristic impedance of the feedthroughs. The beam power dissipated in different ports is shown by the blue curve in Fig. 5. The ports with

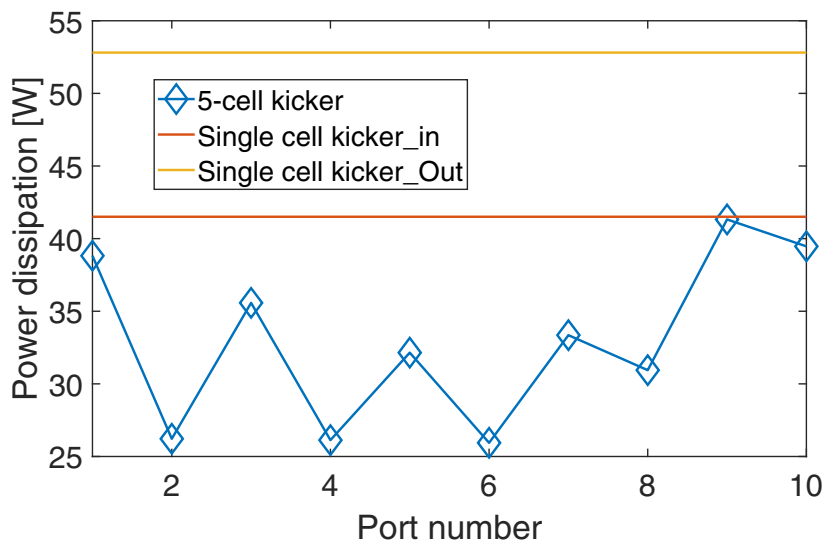

FIG. 5. The blue curve represents the beam power transport through different feedthrough ports of the five-cell kicker. The orange and yellow curves represent that transport through the input and output ports of the single cell kicker.

odd numbers correspond to the upstream ports of the kicker cells, which will be terminated by the matched loads. The ports with even numbers correspond to the downstream ports, which will be connected to the pulsers. We can observe that the power dissipated to the downstream ports is always lower than that to the upstream ports. By comparing the results with the single cell kicker, as shown by the yellow and orange curves, power transport to both the downstream ports and upstream ports is significantly reduced.

\section{Impedance optimization}

In reaching the impedance above, different strategies have been applied to reduce the beam coupling impedance or beam induced heat load. To save the computation time, single cell stripline kickers have been used for the impedance optimizations. To reduce the beam coupling impedance, the distance between the vanes of the beam screen is first reduced. The cross section of the kicker before and after this modification is shown in Figs. 6(a) and 6(b), respectively. While reducing the distance between the vanes, smaller vertical size of the vanes is applied accordingly in order to maintain the field uniformity between the electrodes. On the other hand, the design of the endcovers of the kicker is optimized with smoothed transitions, as shown in Fig. 6(d), instead of hard transitions given in Fig. 6(c).

The effective impedances are compared between different cases. The results are presented in Figs. 7 and 8 . Generally, the loss factor is reduced when decreasing the distance between the vanes, as well as by introducing smooth endcovers. Since more image current can pass through the beam screen and less of them through the electrodes in the situation of closer vanes, the impedance contribution from the cavity structure formed by the electrodes and the beam screen can be mitigated, and less 


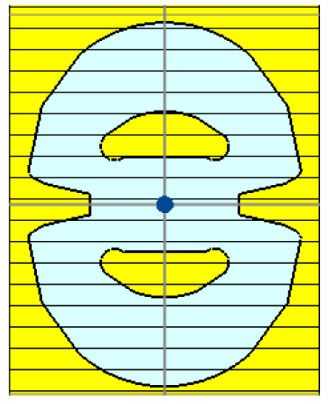

(a)

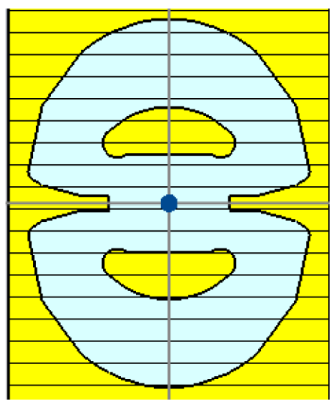

(b)

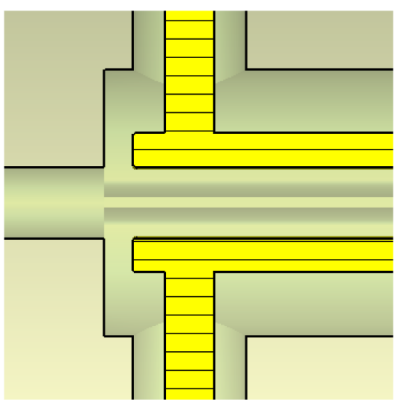

(c)

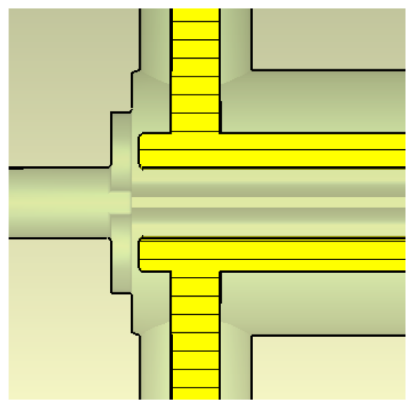

(d)

FIG. 6. Schematic view of the strategies used in the impedance optimizations. (a) and (b) show the transverse cross section of the kicker with wide and closer vanes, respectively. (c) and (d) show hard and smooth transition design of the endcover, respectively.

parasitic losses will occur during the beam passage. The reduction of loss factor with smooth endcover can be explained by the fact that the impedance is directly related to the volume of the cavity structure. By analyzing the power dissipation in the kicker, we found that, with closer vanes, more beam energy will be dissipated on the beam screen, and less on the electrodes, as shown in Fig. 9.

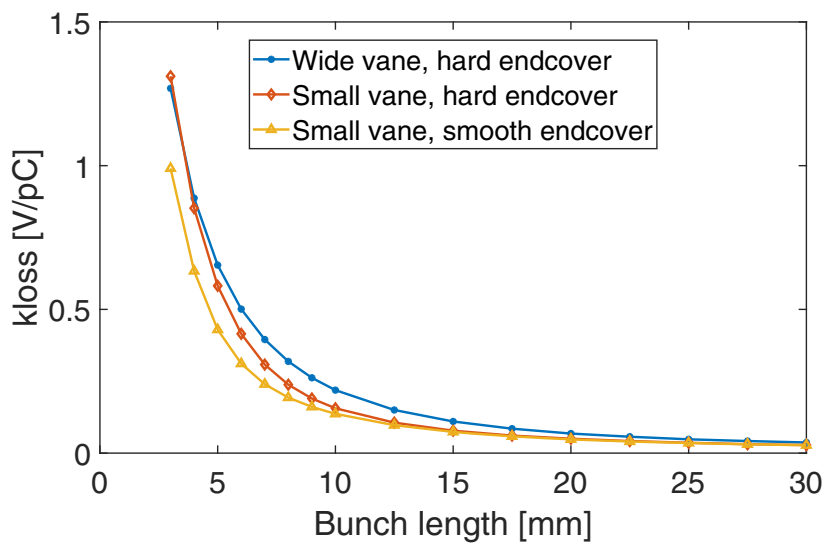

FIG. 7. Dependence of the loss factor on the rms bunch length.

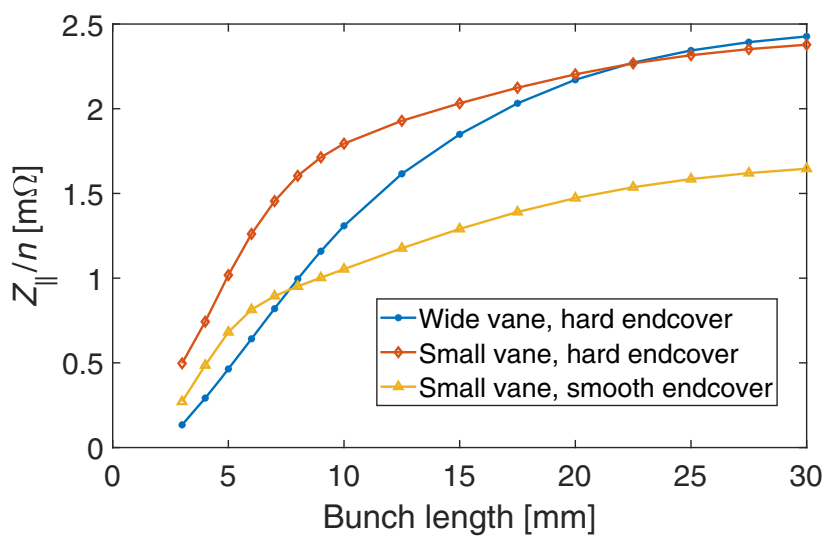

FIG. 8. Dependence of the longitudinal effective impedance on the rms bunch length.
The plateaus between 0.5 and $1.5 \mathrm{~ns}$ correspond to the power losses when the beam passes through the electrodes. Similarly, the power transport from the beam through the feedthroughs is also significantly reduced with closer vanes.

For the longitudinal effective impedance, the cases with closer vanes exhibit higher impedances at short bunch lengths and lower impedances at long bunch lengths. Meanwhile, the impedance is reduced significantly by introducing smooth endcovers. However, less gain is obtained in the transverse case. The vertical effective impedance is reduced by less than $10 \%$ through these optimizations.

\section{BEAM COUPLING IMPEDANCE MEASUREMENTS}

To characterize the impedance of the five-cell stripline kicker experimentally, a campaign of coaxial wire measurements has been launched. The coaxial wire method has been widely used for the impedance bench measurements of the accelerator components [25-34]. The coaxial transmission line formed by the device under test (DUT) and the thin metallic wire on its beam axis are regarded as a

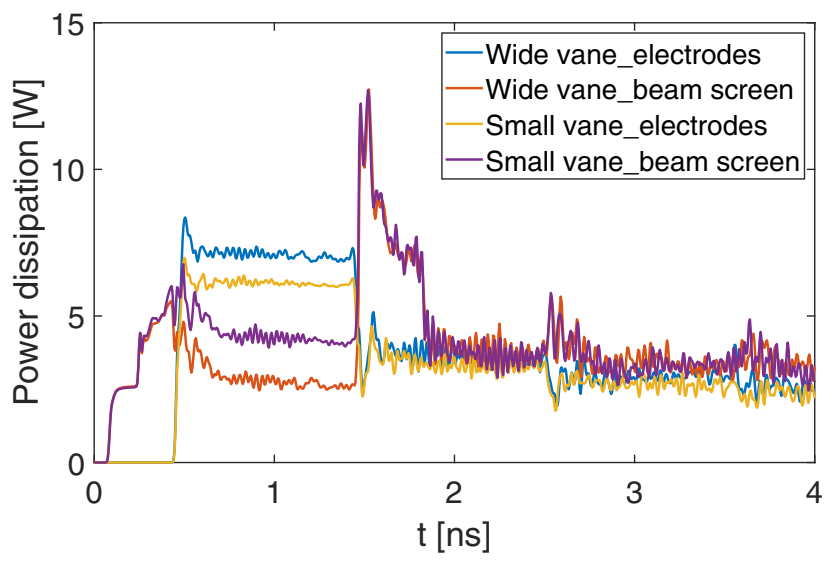

FIG. 9. Power dissipation on the electrodes and the beam screen during the bunch passage with bunch charge of $1 \mathrm{nC}$. 
two-port network. The scattering coefficients of the network are then measured with a vector network analyzer (VNA).

Generally, the impedance mismatch between the DUT and the external circuit can introduce trapped modes and uncertainties to the measured impedances. Matching resistors and taper transitions are commonly used for the impedance matching [32,35-38]. Since the matching resistors are normally frequency dependent, the first method is often used for the bench measurements with frequency below $1 \mathrm{GHz}$. However, in HEPS, the bunch length varies from several millimeters to several centimeters, which relies on the setting of the harmonic rf and bunch lengthening introduced by the impedance or the intrabeam scattering [1]. Therefore, a much wider frequency range is of interest and taper transitions are adopted for the impedance matching.

\section{A. Coaxial wire measurement setup}

During the bench measurements, taper transitions from the stripline kicker to the ring beam pipe with diameter of $22 \mathrm{~mm}$ are included. Therefore, the impedance measured includes contributions from both the stripline kicker and the beam pipe transitions, which as a whole are defined as the DUT. Two drift tubes with diameter of $22 \mathrm{~mm}$ and length of $200 \mathrm{~mm}$ are appended to both ends of the kicker module in order to reduce the reflection from the side ports. A wire made of copper with diameter of $0.8 \mathrm{~mm}$ has been used as the inner conductor. This determines that the cutoff frequency of the measurement setup for a clean transverse electromagnetic (TEM) transmission is around $8 \mathrm{GHz}$. The characteristic impedance of the TEM line at the drift tube is approximately $200 \Omega$. Since the coaxial cables and VNA have characteristic impedances of $50 \Omega$, the large impedance mismatch can induce ripples above the real impedance. To mitigate the effects, taper transitions with aspect ratio of 7 are added at both ends of the DUT.

Since the entire structure has a length of approximately 2 meters and a small gap of $10 \mathrm{~mm}$ between the two parallel electrodes, the effect of sag [25] and centralization of the conducting wire can play significant roles with respect to the accuracy of the coaxial measurements. To solve this issue, a fasten block with ceramic has been designed for the wire stretching and center holding. The inner conductor can thus be stretched and fastened before it is soldered to the pin connectors. Machinable glass ceramic with low permittivity of 6 is adopted for better impedance matching. The CAD drawing of stretching the inner conductor with the fasten block together with the matching section and the soldering panel is shown in Fig. 10; this will then be connected to the VNA with coaxial cables.

The dimensions of the ceramic block have been optimized to reduce its influence on the impedance matching. The transmission of the matching section shown in Fig. 10 is simulated with CST. At frequencies below $7 \mathrm{GHz}$, the

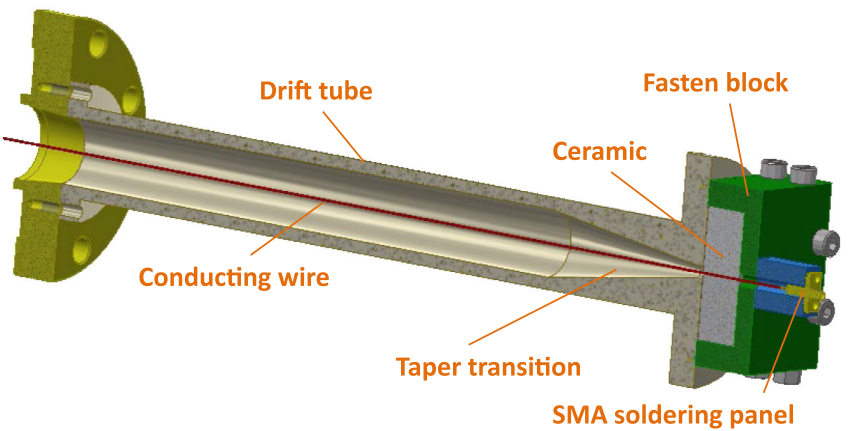

FIG. 10. The CAD drawing of the matching section and mounting of the wire with the ceramic fasten block.

transmission is comparable with that of the case without ceramic block. However, at frequencies above $7 \mathrm{GHz}$, acceptable degradation of the performance has been observed with the ceramic block.

The proposed method is then applied for the bench measurements of the beam coupling impedance of the fivecell stripline kicker. Ten cables, each of which is terminated by a high voltage (HV) attenuator and a $50 \Omega$ load from the VNA calibration kit, are connected to the upstream feedthroughs of the kicker. Here, three different brands of attenuators [39-41] are presently being used for the coaxial wire measurements. Meanwhile, another ten high quality $50 \Omega$ loads are directly connected to the downstream feedthroughs of the kicker to approximate the termination of the pulsers.

\section{B. Benchmark between measurements and simulations}

The S-parameters are measured using the Keysight E5071C 4-port VNA, which has a frequency range of $300 \mathrm{kHz}-20 \mathrm{GHz}$. The transmission $S_{21}$ is measured with frequency resolution of $400 \mathrm{kHz}$. The characteristic impedance along the coaxial transmission line formed by the wire and the stripline kicker is first measured using time domain reflectometry (TDR). This can be used to identify the origin

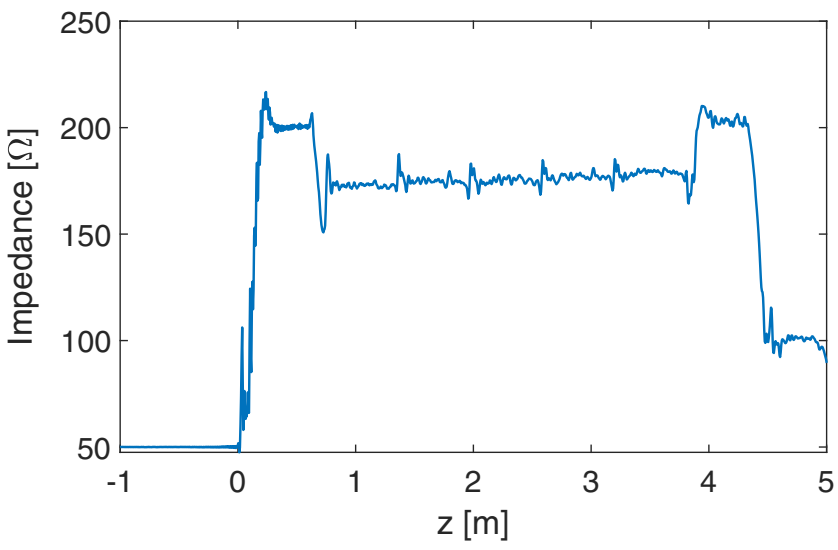

FIG. 11. The characteristic impedance with distance along the coaxial transmission line measured with TDR. 


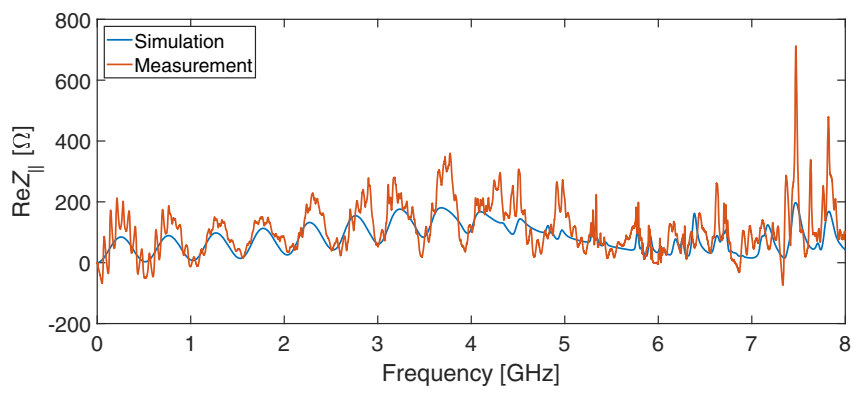

(a)

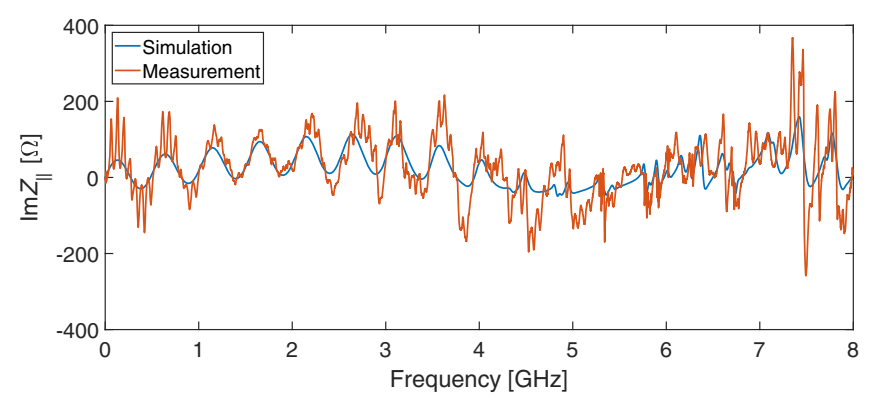

(b)

FIG. 12. The real part (a) and imaginary part (b) of the longitudinal impedance measured by the coaxial method, and compared with simulations.

of the impedance sources, as well as to judge whether the conducting wire is well stretched. The characteristic impedance with distance along the coaxial transmission line is shown in Fig. 11.

Two plateaus of $200 \Omega$ correspond to the drift tubes with diameter of $22 \mathrm{~mm}$, and the small peaks in between represent the impedance mismatch generated by the gap between the adjacent kicker cells, which are also the main source of the impedances. The characteristic impedance at the electrodes is approximately $170 \Omega$, which can be well estimated by the analytical expression [25]:

$$
Z_{c}=60 \ln \left(1.27 \frac{D}{d}\right)=166 \Omega,
$$

where $D$ is the full gap between the parallel electrodes, and $d$ is the diameter of the coaxial wire. The TDR shows no apparent impedance reduction due to the sag of the wire, which means that the wire is well stretched.

Two independent measurements of the transmission coefficient $S_{21}$ of the DUT and a smooth reference tube are carried out to determine the longitudinal beam coupling impedance. The real and imaginary parts of the longitudinal impedance are shown in Fig. 12. The measurement exhibits good frequency resolution of the resonances formed by the electrodes and the beam screen. The residual impedance mismatch between the DUT and measurement network results in reflections, as shown by the ripples every $67 \mathrm{MHz}$. The results are also compared with those obtained

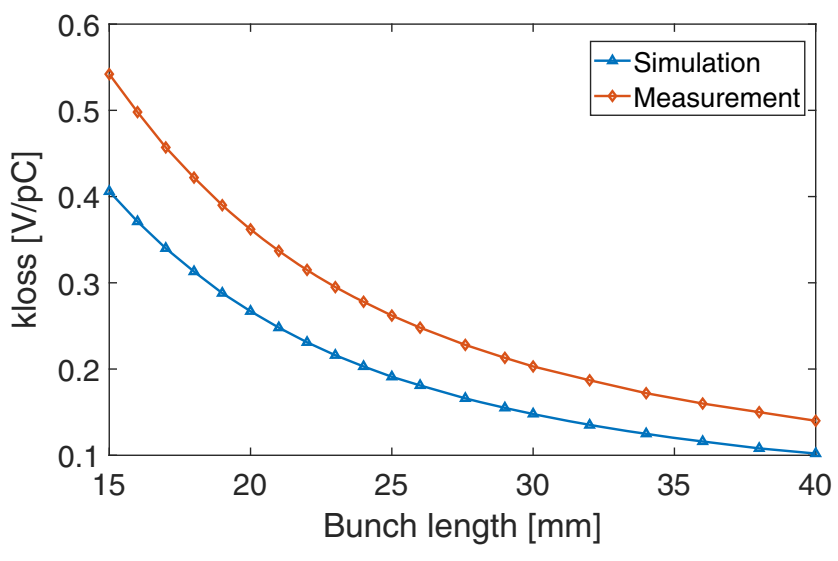

FIG. 13. The loss factor as a function of rms bunch length obtained from coaxial wire measurement and wakefield simulations.

from the wakefield simulations. The measurements show satisfactory agreement with the numerical estimations. This means that the simplified model used in the simulations provides good enough representation of the real impedance characteristic of the kicker. The considerably poor agreement of the impedance amplitude at certain frequencies is probably due to the nonideal properties of the feedthroughs.

With the impedance spectrum given in Fig. 12, the longitudinal effective impedance $Z_{\|} / n$ and loss factor $k_{\text {loss }}$ are calculated with different bunch lengths, as shown in Figs. 13 and 14. The loss factor and longitudinal effective impedance from the measurements are approximately $35 \%$ and $25 \%$ higher than those obtained from the wakefield simulations, respectively.

\section{Influence of the terminations}

In the simulations, all the feedthroughs are treated as ideal coaxial lines with waveguide ports, so that there will be no field reflections from the feedthroughs or

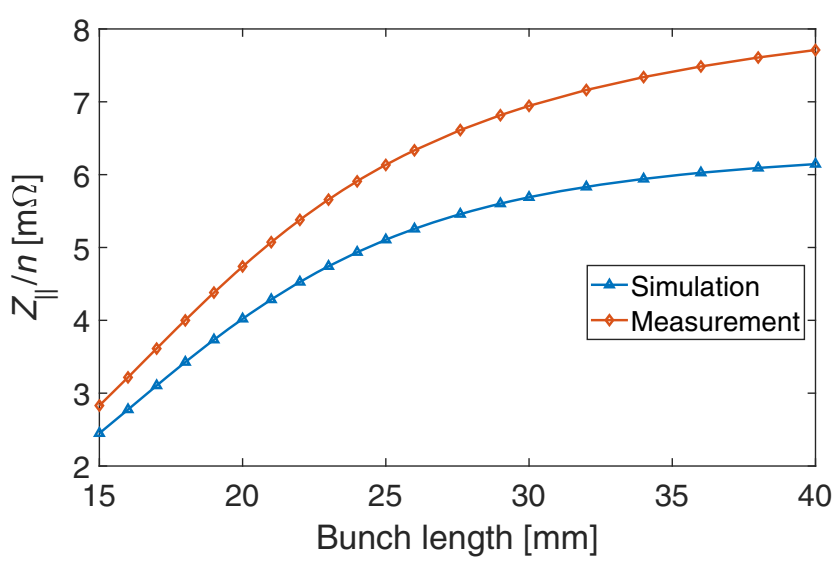

FIG. 14. The longitudinal effective impedance as a function of rms bunch length obtained from coaxial wire measurement and wakefield simulations. 


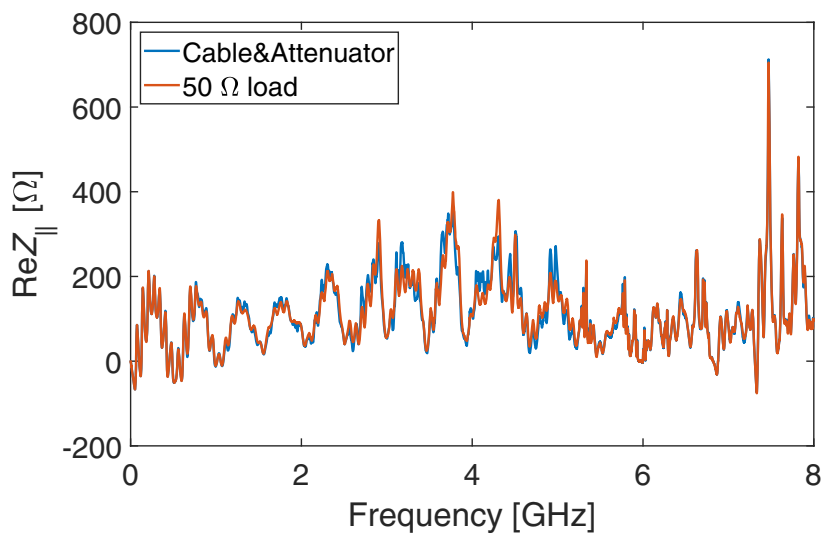

FIG. 15. The real part of the longitudinal impedance measured with different terminations on the output ports.

terminations. However, this is not true for the real case. On the one hand, the feedthroughs are not perfectly matched in order to handle both high voltage and high vacuum. On the other hand, there can always be reflections from the terminations. It is especially difficult to characterize the impedance of the external circuit. Therefore, the influences of different terminations on the beam coupling impedance are investigated experimentally.

First, with the input ports terminated by the high quality $50 \Omega$ loads, the influences of the impedance mismatch from the cables and the HV attenuators are tested. The impedances measured with the output ports terminated by high quality $50 \Omega$ loads directly or through cables and HV attenuators are presented in Fig. 15. Excellent agreement has been reached for these two cases. To better understand the results, separate measurements on the cables and attenuators alone are carried out. Up to $8 \mathrm{GHz}$, the impedance varies between 10 and $250 \Omega$, so that field reflections are expected from the nonperfect matching from the cables and loads. However, further measurements, with two ports of the VNA connecting to the coaxial wire and another port connecting to one of the feedthrough ports, show that the transmissions from the coaxial wire to the feedthroughs, or vice versa, are mainly below $-15 \mathrm{~dB}$. This explained why the nonideal of the cables and actual loads has limited influence on the beam coupling impedance.

Then, with the output ports terminated by the high quality $50 \Omega$ loads through cables and HV attenuators, the influences of different terminations on the input ports are investigated. The impedance measured with the input ports terminated by the $50 \Omega$ loads, open loads and short loads from the VNA calibration kit are presented and compared in Fig. 16. With open loads, the impedance shows strong resonances with increased Q in the frequency range from 2 to $5 \mathrm{GHz}$. This is enhanced for the case terminated with short loads. Frequency shift of the resonances can also be noticed among different cases. To estimate the influences of these divergences, the effective impedance and the loss factor for different cases are calculated with different bunch

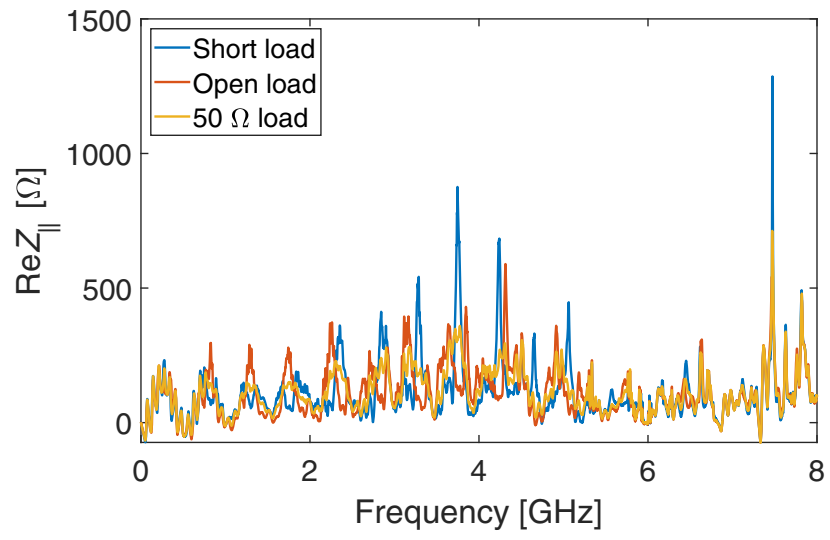

FIG. 16. The real part of the longitudinal impedance measured with different terminations on the input ports.

lengths. The results show that the variation of the loss factor and the broadband effective impedance are less than $2 \%$ with different terminations.

\section{CONCLUSIONS}

Important issues in the design of a stripline kicker are the beam coupling impedance and the heat load in the kicker due to the parasitic power loss of the beam. A new five-cell stripline kicker is proposed in HEPS in order to conserve longitudinal space as well as to reduce the beam coupling impedances. Comprehensive studies have been undertaken to characterize the beam coupling impedance of the fivecell stripline kicker.

The results show that both the longitudinal and transverse impedances of the five-cell stripline kicker present TEM-mode-like resonances below $\sim 4 \mathrm{GHz}$. The impedance behavior at this frequency range is identical to that of the single cell kickers, since the electromagnetic field generated by the beam is more localized. However, at frequencies above $4 \mathrm{GHz}$, the impedances are remarkably reduced in comparison to five, individual, single cell kickers, due to the mitigation of the resonant effect from the interaction among different kicker cells. The advantages of the multicell kicker in reducing the effective impedances are also presented. Both the longitudinal and transverse effective impedances are largely reduced with the five-cell kicker in a wide bunch length interval, from 3 to $30 \mathrm{~mm}$.

With a Gaussian test bunch of rms bunch length of $3 \mathrm{~mm}$, the heat load dissipations due to the beam parasitic power loss in the five-cell stripline kicker are studied in detail. The power losses on the electrodes and beam screen are less than half of those in a single cell kicker. Meanwhile, the beam power transport through the feedthroughs is also significantly reduced compared to the single cell kickers.

Strategies to further reduce the beam coupling impedances or the beam induced heat load of the stripline kicker are also investigated. By reducing the distance between the vanes on the beam screen, more beam power will be 
dissipated on the beam screen, and less on the electrodes. This is important for a kicker design because the electrodes normally face difficulty in handling heating issues. Meanwhile, the beam power transport through the feedthroughs is also significantly reduced with closer vanes. In addition, the endcover of the kicker is optimized with smoothed transitions. This is mainly beneficial in reducing the longitudinal effective impedance.

On the other hand, in order to characterize the impedance of the stripline kicker experimentally, as well as to identify the influence of the nonideal matching from the feedthroughs and the terminations, coaxial wire measurements have been carried out. A satisfactory agreement has been reached between the bench measurements and the wakefield simulations up to $8 \mathrm{GHz}$. The influence of different terminations on the beam coupling impedance is investigated. The results show that the nonideal of the cables and actual loads has limited influence on the beam coupling impedance, and we obtain higher resonances in the range of $2-5 \mathrm{GHz}$ with open or short loads on the input ports. In addition, in order to hold and stretch the conducting wire at the center of the small beam pipe, a novel fasten block design with ceramic is proposed and tested during the measurements. This can be quite useful for the coaxial wire measurements of rather long structures with small apertures.

\section{ACKNOWLEDGMENTS}

The authors would like to thank the HEPS accelerator team for their support and discussions. The work is supported by the National Natural Science Foundation of China (Grants No. 11775239 and No. 11675194), and the Youth Innovation Promotion Association CAS.

[1] Y. Jiao et al., The HEPS project, J. Synchrotron Radiat. 25, 1611 (2018).

[2] Advanced Photon Source Upgrade Project Preliminary Design Report, Argonne National Laboratory, Report No. APSU-2.01-RPT-002, 2017.

[3] L. Farvacque et al., A low emittance lattice for the ESRF, in Proceedings of the 4th International Particle Accelerator Conference, IPAC2013, Shanghai, China, 2013 (JACoW, Shanghai, China, 2013), pp. 79-81.

[4] C. Steier et al., Design progress of ALS-U, the soft x-ray diffraction limited upgrade of the advanced light source, in the 10th International Particle Accelerator Conference (IPAC'19), Melbourne, Australia, 2019 (JACoW, Melbourne, Australia, 2019), TUPGW097.

[5] S. C. Leemann, A. Andersson, M. Eriksson, L.-J. Lindgren, E. Wallen, J. Bengtsson, and A. Streum, Beam dynamics and expected performance of Sweden's new storage-ring light source: MAX IV, Phys. Rev. ST Accel. Beams 12, 120701 (2009).

[6] L. Emery and M. Borland, Possible long-term improvements to the Advanced Photon Source, in Proceedings of the 2003 Particle Accelerator Conference, Portland, OR (IEEE, New York, 2003), pp. 256-258, TOPA014.

[7] S. C. Leemann, Pulsed sextupole injection for Sweden's new light source MAX IV, Phys. Rev. ST Accel. Beams 15, 050705 (2012).

[8] M. Aiba, M. Boege, F. Marcellini, A. S. Hernandez, and A. Streum, Longitudinal injection scheme using short pulse kicker for small aperture electron storage rings, Phys. Rev. ST Accel. Beams 18, 020701 (2015).

[9] S. Jiang and G. Xu, On-axis injection scheme based on a triple-frequency rf system for diffraction-limited storage rings, Phys. Rev. Accel. Beams 21, 110701 (2018).

[10] C. Steier, A. Anders, T. Luo, T. Olider, C. Pappas, S. De Santis, C. Sun, C. Swenson, and W. Waldron, On-axis swap-out R\&D for ALS-U, in Proceedings of IPAC2017, Copenhagen, Denmark (JACoW, Copenhagen, Denmark, 2017), WEPAB103.

[11] D. Alesini, S. Guiducci, F. Marcellini, and P. Raimondi, Design, test, and operation of new tapered stripline injection kickers for the $e^{+} e^{-}$collider DAФNE, Phys. Rev. ST Accel. Beams 13, 111002 (2010).

[12] C. Belver-Aguilar, A. Faus-Golfe, F. Toral, and M. J. Barnes, Stripline design for the extraction kicker of Compact Linear Collider damping rings, Phys. Rev. ST Accel. Beams 17, 071003 (2014).

[13] J. H. Chen, H. Shi, L. Wang, Z. Duan, N. Wang, L. H. Huo, G. W. Wang, X. L. Shi, and P. Liu, Strip-line kicker and fast pulser R\&D for the HEPS on-axis injection system, Nucl. Instrum. Methods Phys. Res., Sect. A 920, 1 (2019).

[14] W. Liu, L. Shang, W. Song, F. Shang, and Z. Sun, Development of a stripline kicker for the Hefei Advanced Light Facility, Nucl. Instrum. Methods 961, 163670 (2020).

[15] T. Naito, S. Araki, H. Hayano, K. Kubo, S. Kuroda, N. Terunuma, T. Okugi, and J. Urakawa, Multibunch beam extraction using the strip-line kicker at the KEK Accelerator Test Facility, Phys. Rev. ST Accel. Beams 14, 051002 (2011).

[16] B. I. Grishanov, F. V. Podgorny, J. Rummler, and V. D. Shiltsev, Very fast kicker with high repetition rate for accelerator applications, Nucl. Instrum. Methods Phys. Res., Sect. A 396, 28 (1997).

[17] T. Naito, H. Hayano, M. Kuriki, N. Terunuma, and J. Urakawa, Development of a 3 ns rise and fall time stripline kicker for the International Linear Collider, Nucl. Instrum. Methods Phys. Res., Sect. A 571, 599 (2007).

[18] S. De Santis et al., Injection/extraction kicker for the ALS-U project, in Proceedings of the IPAC2014, Dresden, Germany (JACoW, Dresden, Germany, 2014), WEPRO016.

[19] C. Yao et al., Development of fast kickers for the APS MBA upgrade, in Proceedings of the IPAC2015, Richmond, VA (JACoW, Richmond, VA, USA, 2015), pp. 3286-3288, WEPTY014.

[20] H. Shi, J. H. Chen, L. Wang, N. Wang, L. H. Huo, G. W. Wang, P. Liu, and X. L. Shi, Development of a 750-mmlong stripline kicker for HEPS, Radiat. Detection Technol. Methods 2, 47 (2018). 
[21] L. Wang et al., A $300 \mathrm{~mm}$ long prototype strip-line kicker for the HEPS injection system, in Proceedings of IPAC2019, Melbourne, Australia (JACoW, Melbourne, Australia, 2019), THPRB026.

[22] CST STUDIO SUITE website: http://www.cst.com.

[23] A. Blednykh, B. Bacha, G. Bassi, C. Hetzel, B. Kosciuk, D. Padrazo, T. Shaftan, V. Smaluk, and T. Ha, Beam impedance and heating analysis of the diagnostic stripline, Nucl. Instrum. Methods Phys. Res., Sect. A 963, 163729 (2020).

[24] A. W. Chao and M. Tigner, Handbook of Accelerator Physics and Engineering (World Scientific, Singapore, 1998), p. 208.

[25] T. Kroyer, F. Caspers, and E. Gaxiola, Longitudinal and transverse wire measurements for the evaluation of impedance reduction measures on the MKE extraction kickers, CERN Report No. AB-Note-2007-028.

[26] M. Sands and J. Rees, A bench measurement of the energy loss of a stored beam to a cavity, SLAC-Report No. PEP-95, 1974.

[27] H. Hahn, Validity of coupling impedance bench measurements, Phys. Rev. ST Accel. Beams 3, 122001 (2000).

[28] V. G. Vaccaro, Coupling impedance measurements: An improved wire method, Report No. INFN/TC-94/023, Sezione di Napoli, 1994.

[29] G. Nassibian and F. Sacherer, Methods for measuring transverse coupling impedances in circular accelerators, Nucl. Instrum. Methods 159, 21 (1979).

[30] F. Caspers, Beam impedance measurements using the coaxial wire method, CERN Report No. PS/88-59 (AR/OP), 1988.
[31] F. Caspers, in Handbook of Accelerator Physics and Engineering, edited by A. W. Chao and M. Tigner (World Scientific, Singapore, 1998), p. 570.

[32] L. S. Walling, D. E. McMurry, D. V. Neuffer, and H. A. Thiessen, Transmission line impedance measurements for an advanced hadron facility, Nucl. Instrum. Methods Phys. Res., Sect. A 281, 433 (1989).

[33] H. Hahn and F. Pedersen, On coaxial wire measurements of the longitudinal coupling impedance, Report No. BNL 50870, 1978.

[34] E. Jensen, An improved log-formula for homogeneously distributed impedance, Report No. PS/RF/Note 2000-001, 2000.

[35] N. Biancacci, F. Caspers, J. Kuczerowski, E. Metral, N. Mounet, B. Salvant, A. Mostacci, O. Frasciello, and M. Zobov, Impedance simulations and measurements on the LHC collimators with embedded beam position monitors, Phys. Rev. Accel. Beams 20, 011003 (2017).

[36] J. G. Wang and S. Y. Zhang, Coupling impedance measurements of a model fast extraction kicker magnet for the SNS accumulator ring, Nucl. Instrum. Methods Phys. Res., Sect. A 522, 178 (2004).

[37] J. G. Wang and S. Y. Zhang, Measurement of coupling impedance of accelerator devices with the wire-method, Nucl. Instrum. Methods Phys. Res., Sect. A 459, 381 (2001).

[38] U. Niedermayer, L. Eidam, and O. Boine-Frankenheim, Analytic modeling, simulation and interpretation of broadband beam coupling impedance bench measurements, Nucl. Instrum. Methods Phys. Res., Sect. A 776, 129 (2015).

[39] http://www.fidtechnology.com/.

[40] https://barthelectronics.com/.

[41] http://www.shx-sh.com/. 